\title{
Atelier de théâtre : Le marchand de Venise
}

\section{(2) OpenEdition}

1 Journals

\section{Édition électronique}

URL : http://journals.openedition.org/shakespeare/530

DOI : $10.4000 /$ shakespeare.530

ISSN : 2271-6424

Éditeur

Société Française Shakespeare

\section{Édition imprimée}

Date de publication : 1 novembre 1989

Pagination : 171-175

\section{Référence électronique}

"Atelier de théâtre : Le marchand de Venise », Actes des congrès de la Société française Shakespeare [En ligne], 6 | 1989, mis en ligne le 01 janvier 2007, consulté le 01 mai 2019. URL : http://

journals.openedition.org/shakespeare/530; DOI : 10.4000/shakespeare.530 


\section{SOCIETE FRANCAISE SHAKESPEARE}

\section{Actes du Congrès - 1984}

\section{LIEU ET TEMPS}

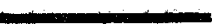

Directeur de la publication Jean FUZIER 


\section{Atelier de Théâtre Le Marchand de Venise}

Avec la participation de :

- Susan Todd, qui dirige l'atelier. Elle a déjà dirigé de nombreuses mises en scène dans les théâtres régionaux d'Angleterre. Elle a été assistante de John Barton à la Royal Shakespeare Company. Elle a fait du travail expérimental à Londres. Deux de ses mises en scène sont actuellement en cours : Body and Soul à la Royal Court Theatre, et Pax qui est en tournée en Grande-Bretagne.

- Jennie Stoller, son travail inclut le rôle de Helena dans The Dream monté par Peter Brook. Elle a participé à la tournée internationale de la Royal Shakespeare Company. Elle a joué le rôle de Mrs Kendle dans The Elephant Man au National Theatre. Elle a pris part à une tournée aux Etats-Unis avec la R.S.C. dans le rôle de Portia (le Marchand de Venise).

- Roger Allam, qui a joué Mercutio dans Romeo et Juliette à Ștratford l'été dernier, Kent dans Richard III et Oberon et Theseus dans le Songe d'une Nuit d'Eté. Il joue dans Today de Robert Holman et aussi dans The Party de Trevor Griffits. 
Susan Todd: Dans cet atelier, nous allons explorer quelques idées à travers trois passages.

Avant de relire Le Marchand de Venise, je pensais que cette pièce était injouable au $\mathrm{XX}^{\mathrm{e}}$ siècle car elle est antisémite. On ne peut pas contourner cet obstacle, et c'est un grand problème pour la conscience contemporaine. Le personnage de Shylock a complètement envahi la pièce de nos jours. Mais nous sentons un changement de perspective. Nous avons pensé que nous pourrions envisager un autre aspect, du moins pour commencer : quelle est la signification de la notion de lien dans la pièce? Toutes sortes de liens, d'engagements existent entre les individus, qui peuvent aller jusqu'au plus rigide et au plus sinistre (entre Shylock et Antonio). Personnellement, je suis très intéressée par le personnage de Portia. En ne considérant que la question juive, on perd de vue le fait que Portia occupe une place centrale dans la pièce. Elle parvient à établir une sorte d'équilibre en ce qui concerne cette notion de lien. Elle-même est liée à son père défunt. Bien que son exigeance soit pour elle une source de chagrin, elle s'y tient. Au cours de la pièce, elle montre à Bassanio comment établir et développer un véritable lien avec elle conduisant au mariage. De même, elle essaie d'enseigner à Shylock ce que serait un lien acceptable avec d'autres êtres humains. Je n'ai jamais vu de mise en scène de cette pièce qui accorde une place centrale au cheminement de Portia, incluant Bassanio (une mise en scène récente de John Barton développait intelligemment la relation entre Bassanio et Antonio, mais Portia n'existait pas).

* Premier extrait: Le premier lien qui apparait dans la pièce est celui qui existe entre Antonio et Bassanio - I, i, v. 114-fin scène. Jennie joue le rôle d'Antonio, Roger, celui de Bassanio.

Cette scène d'ouverture est très importante puisqu'elle montre la signification d'un lien entre deux individus. Psychiquement Antonio fait fonction de père, en termes de statut et de pouvoir, et Bassanio de fils. C'est une relation très tendue, comme si Shakespeare démontrait qu'une relation avec un parent pouvait être suffocante (voir Jessica et Shylock).

Ce passage est joué plusieurs fois afin de saisir les besoins émotionnels et les rythmes des personnages. 
1 - Bien que la structure du texte soit d'une très grande douceur, Bassanio doit s'en défendre. Il doit apparaître dans un état peu cohérent, et exagérer ses doutes.

Antonio pourrait être chaleureux, généreux, indulgent envers cet enfant errant ou ce jeune frère (bien qu'ils puissent avoir le même âge) à qui il arrive toujours des ennuis.

2 - En cherchant un ton plus triste: ils comprennent l'un et l'autre que le voyage vers Portia signifie pour eux une séparation. Il faut sentir le changement de ton dans le personnage d'Antonio.

3 - Bassanio doit rester incohérent. Il ne sait pas ce qu'il va dire ensuite, il n'est nullement calculateur, il se laisse emporter.

Antonio lutte en son for intérieur. Il ne supporte pas de voir Bassanio malheureux. Il se procurera l'argent dont il a besoin coûte que coûte. Il et homme à risquer le tout pour le tout.

4 - «... therefore go forth " (v. 180), en accentuant la pause.

5 - Changement de rôles : Jennie est Bassanio, Roger, Antonio. Roger a repris ces quelques mots avec une nuance d'ironie.

6 - Travail sur la respiration toujours sur ces quelques mots. Il faut amplifier les transitions afin de ne pas perdre sa subtilité même devant un large auditoire.

* Dans le deuxième extrait, Portia et Bassanio se déclarent mutuellement leur attachement : III, 2, v. 1-41

Une scène très directe dans laquelle Portia comprend avec tristesse que la promesse qui la lie à la volonté de son père peut lui faire perdre quelqu'un qui lui est particulièrement cher. La langue est très raffinée, c'est une déclaration très fragile.

Ils ont suffisamment échangé de propos pour qu'elle s'exprime très librement. Mais Bassanio n'est pas arrivé depuis bien longtemps (quelques heures), aussi on doit sentir une certaine urgence dans leur entretien.

Susan place le décor : la terrasse, la maison. Elle insiste sur l'importance du cheminement de Bassanio, de l'extérieur vers la terrasse. Il faut imaginer une distance de 500 mètres environ à travers un jardin et visualiser la porte derrière laquelle sont les coffrets.

1 - Bassanio doit prendre son temps pour se donner du courage et bien considérer l'importance de sa démarche. 
2 - Travail sur les déplacements des deux acteurs.

3 - On doit sentir le dilemme de Portia qui veut faire comprendre à Bassanio la vraie qualité de ses sentiments. Mais il y a une différence dans le fait d'aller vers les pensées de Bassanio ou de les solliciter vers elle.

$4-v .28$ Il y a un changement distinct de ton: il devient plus léger, elle ne sait pas encore que sa plaisanterie cache une réalité.

5 - Portia doit utiliser le déplacement de Bassanio vers elle pour alléger : " $O$ ! these naughty times » (v.18) afin de parvenir à un ton presque comique et permettre à Bassanio d'assumer le choix effrayant qu'il doit faire.

6 - A partir du vers 18 , Bassanio se rapproche le plus près possible de Portia.

7 - On doit sentir une ambivalence : Portia peut éventuellement perdre Bassanio s'il fait le mauvais choix, mais à la fois, les spectateurs doivent rester assurés qu'il va sûrement choisir le bon coffret.

8 - Echange de regards complices entre Bassanio et Portia.

9 - Paradoxalement, il faut montrer que le ton s'allège sur le mot "treason" (v. 27). Il et très difficile de trouver un équilibre entre un ton léger ou plus sérieux.

*Troisième extrait: une séquence où apparaît Shylock III, 1 v. 25-fin scène

La situation est insupportable de cruauté, mais il est très difficile de ne pas se laisser aller à de la sentimentalité. Les sentiments de Shylock passent très vite de l'envie à la rage, à la colère profonde... On doit constamment sentir la présence de Jessica. Imaginez-la morte dans son cercueil, les ducats à côté d'elle. Montrez la force de Shylock qui la tue en paroles.

Jennie joue Tubal, Roger est Shylock.

1 - Shylock ne doit pas être sentimental, mais il est très émouvant. Ne pas oublier qu'il parle de vengeance.

2 -Montrer le déchirement de Shylock : il perd tout en un éclair. Il ne fait que hurler sa douleur, tout sort à la fois. Il ne pense pas encore à sa vengeance, il souffre trop. Tout s'effondre autour de lui. 
3 - Faire sentir la rupture de ton à la fin de la scène : « ... fetch me an officer" (v. 134). A ce moment-là, il a pris la décision de se venger et il va s'y tenir.

4 - Ne pas rater la très belle sortie de Shylock. Laurence Olivier sortait de scène en criant. Il était absolument impossible de croire à la scène suivante. On ne peut obtenir une mise en scène satisfaisante si on ne la concentre que sur Shylock et ses grandes souffrances. Elles étaient présentes du temps de Shakespeare, mais la pièce portait surtout sur Portia, Antonio et Bassanio. 